\section{Superiority of Carboplatin-Based Doublets to Cisplatin-Based Doublets among Medicare Patients Aged 70 and Older with Advanced Lung Cancer}

\section{Abstract}

Background: Some evidence suggests that cisplatin-based chemotherapy doublet regimens are more effective than the less toxic carboplatin-based doublet regimens for patients with advanced non-small cell lung cancer (NSCLC), but their effectiveness among elderly cancer patients who are treated in usual care settings is not known.

Methods: We identified 13,406 elderly Medicare patients who were diagnosed with stage IV NSCLC between the years 1995-2007 in SEER regions and treated with either a cisplatin-based or a carboplatin-based doublet chemotherapy regimen in the subsequent six months. Using propensity score weighting, we balanced the two treatment cohorts with respect to observable attributes. We then estimated survival and morbidity according to treatment.

Findings: Overall, patients treated with cisplatin-based doublets lived two weeks longer on average than patients treated with carboplatin-based doublets (i.e., 7.4 months vs. 7.0 months, $p=0.05$ ). For patients $>70$ years of age, first-line therapy with cisplatin-based doublet chemotherapy regimens was associated with increased post-treatment morbidity and lack of survival advantage compared to carboplatin-based doublet therapies.

Interpretations: For patients $>70$ years of age first-line therapy with carboplatinbased doublet chemotherapy regimens are preferable to cisplatin-based doublet therapies given the similar survival and lower risk of hospitalization.

Keywords: Medicare; Lung cancer; Effectiveness; Chemotherapy; Health care use; Hospitalizations; Elderly cancer patients; Cisplatin; Carboplatin
Elizabeth Lamont ${ }^{1-3 *}$, Nancy L Keating ${ }^{2-4}$, Christopher Azzoli ${ }^{1,2}$ and Mary Beth Landrum ${ }^{3}$

1 Massachusetts General Hospital Cancer Center, Boston, USA

2 Department of Medicine, Harvard Medical School, Boston, USA

3 Department of Health Care Policy, Harvard Medical School, Boston, USA

4 Section of General Internal Medicine, Department of Medicine, Brigham and Women's Hospital, Boston, USA

Corresponding author: Elizabeth B. Lamont

” ewb.76.Is@gmail.com

Massachusetts General Hospital Cancer Center, Boston, USA.

Tel: 617-894-8978

Citation: Lamont E, Keating NL, Azzoli C, Landrum MB (2018) Superiority of Carboplatin-Based Doublets to Cisplatin-Based Doublets among Medicare Patients Aged 70 and Older with Advanced Lung Cancer. Arch Med. Vol. 10 No.2:4

\section{Introduction}

Lung cancer is the second most common cancer in both men and women in the United States (US), the leading cause of cancer death for men and women, and arguably a disease of the elderly [1]. Of the $>224,000$ American men and women who were diagnosed with lung cancer in 2014 , greater than $70 \%$ were diagnosed at or after age 65.1 Among these 155,000 elderly individuals, more than half were diagnosed with disseminated (i.e., metastatic) disease, which is incurable and often rapidly fatal (i.e., median survival $<12$ months) even with aggressive chemotherapy treatments.
Over the last thirty years, randomized clinical trials (RCTs) have established that treatment with chemotherapy both improves quality of life and extends survival over best supportive care alone in patients with advanced non-small cell lung cancer (NSCLC), which is the most common form of lung cancer in the US [2]. Subsequent research has established that combination chemotherapy with "platinum-based" doublet chemotherapy regimens is superior to single agents or non-platinum-based combination regimens [3-5]. Among platinum-based doublets regimens, there is ambiguity in clinical oncology about which platinum-containing drug (i.e., cisplatin or carboplatin) confers the greatest benefit. Some randomized trials reported no 
survival differences by platinum agent, as did a subsequent metaanalysis [6-9]. However, other randomized trials, meta-analyses and a single-institution retrospective study suggested that at least response rate (and possibly survival) following cisplatinbased regimens is greater than for carboplatin-based regimens [10-12]. Subset analyses of two of these studies based on NSCLC histology showed conflicting results; the single institution retrospective study showed that cisplatin-based regimens were more efficacious for patients with squamous cell histologies but the meta-analysis reported that cisplatin-based regimens were more efficacious for patients with non-squamous cell histologies $[11,12]$. A 2013 Cochrane Review of randomized trials concluded that survivals were equal and thus treatment should be tailored to individual patients, guided by the toxicity profiles of the regimens [12].

There is less ambiguity about differences in post-treatment morbidity. Cisplatin-based regimens are generally associated with more clinically significant toxicity, including nausea, vomiting, ototoxicity, and nephrotoxicity, while carboplatinbased regimens are associated with greater risk of clinically significant thrombocytopenia [13].

Despite the substantial number of existing reports from clinical trials, subset analyses, and meta-analyses that were done to answer the question of whether cisplatin-based regimens are more efficacious than carboplatin-based regimens overall and in several sub-groups of patients, there is a relative paucity of evidence regarding the effectiveness of cisplatin-based vs. carboplatin-based regimens in the nearly 80,000 elderly Medicare patients with NSCLC who are diagnosed and cared for annually in the usual care setting. These patients are typically underrepresented in randomized trials and elderly patients may be more susceptible to increases in certain treatment-related toxicities (e.g., cisplatin-related renal toxicity given normal agerelated loss of nephrons over time, carboplatin-related platelet toxicity given normal age-related delays in bone marrow recovery over time). The existing observational studies of chemotherapy in elderly patients do not answer this specific question either because they have been agnostic to the precise chemotherapy drugs and schedules, or because they studied patients who have arguably poorer baseline health than elderly Medicare patients [14-21].

In an effort to provide useful evidence for decision-making for oncologists and their patients with NSCLC who are elderly, we studied observational data to estimate differences in morbidity and mortality between first-line cisplatin-based and carboplatinbased doublet chemotherapy regimens in elderly Medicare patients with advanced NSCLC who are treated in the usual care setting.

\section{Methods}

\section{Data Sources and Cohort}

The SEER-Medicare dataset is a National Cancer Institute ( $\mathrm{NCl}$ )sponsored cancer patient-level linkage of clinical data collected by the SEER cancer registries with Medicare billing claims. These data are widely used by researchers studying outcomes, clinical epidemiology, and health services factors among older cancer patients [22].

The SEER program collects information regarding the diagnosis and treatment of patients with cancer from population-based cancer registries to monitor trends in incidence and survival. It is estimated that up to $28 \%$ of the American population with cancer is represented in these data [23]. Prior research has shown that, in the aggregate, patients in these registries are demographically representative of the general population [24]. The SEER program reports patient demographics and detailed information about the cancer diagnosis including month and year of diagnosis, site, histology, extent of tumor at diagnosis, initial cancer-directed surgical and radiation therapy, but not chemotherapy. SEER also reports date of death which it obtains from the national death index. Additionally, the SEER data are linked at the patient level to US Census data, which we used to characterize socioeconomic attributes of patients' neighborhoods.

Medicare is a federally sponsored health insurance program administered by the Centers for Medicare and Medicaid Services (CMS) whose beneficiaries include more than $96 \%$ of all US citizens aged 65 and older. CMS maintains billing records of ambulatory, inpatient, home health, hospice, durable Medical Equipment (DME), and other claims for all beneficiaries not enrolled in risk contract health maintenance organizations (HMOs). Importantly, Medicare reimburses for cancer-directed therapies including chemotherapy. It is from Medicare claims that information on elderly SEER patients' chemotherapy use is obtained in studies that utilize the linked SEER-Medicare data.

\section{Elderly Medicare Advanced NSCLC Cohort}

We identified 102,445 patients aged 66 and older who were diagnosed in one of the SEER areas with stage IV NSCLC between the years 1995-2009 and were without a prior history of cancer. By conditioning on age $>66$ years at diagnosis, we ensured at least 12 months of Medicare data prior to patient diagnosis (Medicare claims are available for the patients beginning in 1991 and extending through 2010), which are useful in determining salient baseline patient characteristics reflecting case-mix or medical heterogeneity between treatment groups (see Matching Approach below). We required that the analytic sample be continuously eligible for Medicare Parts A and B and never enrolled in an HMO from the 12 months prior to diagnosis through date of death (or last Medicare claim date for those who did not die during the observation period), have diagnostic confirmation of their cancer, not received experimental therapy, and have at least one Medicare claim in the period 45 days before diagnosis through 195 days after diagnosis to ensure linkage, restricting the sample to 57,932 patients. We evaluated all patients' Medicare claims files in the six months following diagnosis with previously validated methods and identified $57 \%(32,885 / 57932)$ of patients who had received chemotherapy during this period. Of those, $41 \%(13,406 / 32,885)$ of patients received one of the first-line platinum-based doublet chemotherapy regimens of interest (Appendix A) in the ambulatory setting [25]. The distributions of the doublets according to platinum agent are listed in Appendix B. 


\section{Outcome Variables}

We obtained date of death from the Medicare enrollment file and examined overall survival from the date of first chemotherapy claim. We censored observations alive as of December 31, 2010 (the last date with complete vital status data available) or after five years of follow-up. To assess morbidity we examined health care use following initiation of chemotherapy including hospitalization, intensive care unit (ICU) stays, emergency room (ER) visits, home health, skilled nursing facility (SNF) stays, hospice and death in a hospital. The hospital-based health care that elderly Medicare patients with cancer use while they are on chemotherapy trials is strongly associated with their clinical trial measure of post-treatment morbidity [26]. Finally we examined claims for hemodialysis as cisplatin-based regimens are associated with nephrotoxicity. Codes used to ascertain these outcomes are included in Appendix C.

\section{Propensity Score Weighting Approach}

To minimize the bias related to the non-random allocation of multi-agent treatment regimen, we used propensity score weighting to compare patients treated with cisplatin-based regimens with similar patients treated with carboplatin-based regimens. We first fit a logistic regression model to predict receipt of a cisplatin-based versus carboplatin-based regimen as a function of salient baseline covariates, including demographic variables (age, race, sex, marital status, a Medicare poverty indicator, percent of residents with a college degree and median per capita income in patients' census tract); oncologic (i.e., tumor histology, brain metastases), medical comorbidity (i.e., Charlson comorbidity score, history of chronic obstructive pulmonary disease (COPD)), and health care use in the year prior to first chemotherapy treatment (i.e., history of prior ER visit or hospitalization with a primary diagnosis of COPD, supplemental oxygen use (see Appendix D), equipment to improve patients' functional status, (Appendix D), days in a hospital, care from a home health agency (HHA), and admission to a skilled nursing facility (SNF), geographic factors (i.e., SEER registry) and temporal factors (i.e., year of diagnosis)) [27-29].

Covariates were categorized as in Table 1 . We used the model

Table 1 Attributes of patients treated with carboplatin- vs cisplatin-based doublet regimens for metastatic NSCLC before and after propensity score weighting $(\mathrm{N}=13,406)$.

\begin{tabular}{|c|c|c|c|c|c|c|}
\hline Variables & $\begin{array}{l}\text { Carboplatin-based } \\
\text { Doublet } N=12,501\end{array}$ & \multicolumn{2}{|c|}{$\begin{array}{l}\text { Cisplatin-based } \\
\text { Doublet } N=905\end{array}$} & $\begin{array}{l}\text { Carboplatin-based } \\
\text { Doublet } N=12,501\end{array}$ & \multicolumn{2}{|c|}{ Cisplatin-based Doublet $\mathrm{N}=905$} \\
\hline \multicolumn{3}{|c|}{ Age at diagnosis (\%) } & $<0.01$ & & & 0.90 \\
\hline $66-69$ & $28 \cdot 8$ & $35 \cdot 8$ & & $29 \cdot 2$ & $28 \cdot 8$ & \\
\hline $70-74$ & $34 \cdot 3$ & $39 \cdot 8$ & & $34 \cdot 7$ & $32 \cdot 8$ & \\
\hline $75-79$ & $25 \cdot 1$ & $19 \cdot 2$ & & $24 \cdot 7$ & $26 \cdot 2$ & \\
\hline $80-84$ & $9 \cdot 9$ & NR & & $9 \cdot 5$ & $10 \cdot 1$ & \\
\hline $85+$ & 1.9 & NR & & $1 \cdot 8$ & $2 \cdot 1$ & \\
\hline Female (\%) & $42 \cdot 1$ & $35 \cdot 7$ & $<0.01$ & $41 \cdot 7$ & $43 \cdot 3$ & 0.46 \\
\hline \multicolumn{3}{|c|}{ Race (\%) } & 0.27 & & & 0.87 \\
\hline White & $84 \cdot 8$ & $84 \cdot 2$ & & $84 \cdot 7$ & $83 \cdot 9$ & \\
\hline Black & $7 \cdot 3$ & $6 \cdot 7$ & & $7 \cdot 2$ & $7 \cdot 2$ & \\
\hline Hispanic & $3 \cdot 1$ & $2 \cdot 8$ & & $3 \cdot 0$ & $3 \cdot 2$ & \\
\hline Other & $4 \cdot 9$ & $6 \cdot 3$ & & $5 \cdot 0$ & $5 \cdot 8$ & \\
\hline \multicolumn{3}{|c|}{ Marital Status (\%) } & $<0.01$ & & & 0.36 \\
\hline Married & $61 \cdot 7$ & $66 \cdot 2$ & & $62 \cdot 0$ & $60 \cdot 1$ & \\
\hline Single & $6 \cdot 5$ & $7 \cdot 6$ & & $6 \cdot 6$ & $8 \cdot 1$ & \\
\hline Other Single & $31 \cdot 8$ & $26 \cdot 2$ & & $31 \cdot 5$ & $31 \cdot 8$ & \\
\hline State Buy-In (mos) & $1 \cdot 2$ & $1 \cdot 4$ & $0 \cdot 21$ & $1 \cdot 3$ & $1 \cdot 3$ & 0.78 \\
\hline $\begin{array}{l}\% \text { College graduates in } \\
\text { census tract of residence }\end{array}$ & $25 \cdot 1$ & $25 \cdot 5$ & 0.42 & $25 \cdot 1$ & $24 \cdot 7$ & 0.62 \\
\hline $\begin{array}{l}\text { Median Income of census } \\
\text { tract of residence }\end{array}$ & $\$ 49,962$ & $\$ 51,384$ & 0.08 & $\$ 50,044$ & $\$ 49,751$ & 0.76 \\
\hline Missing Census data (\%) & NR & NR & $0 \cdot 17$ & 0.09 & $0 \cdot 12$ & 0.74 \\
\hline \multicolumn{3}{|c|}{ Histology (\%) } & 0.18 & & & 0.91 \\
\hline Adenocarcinoma & $42 \cdot 1$ & $40 \cdot 1$ & & $42 \cdot 0$ & $40 \cdot 3$ & \\
\hline Squamous Cell & $22 \cdot 4$ & $25 \cdot 6$ & & $22 \cdot 6$ & $22 \cdot 4$ & \\
\hline Large Cell & $5 \cdot 1$ & NR & & $5 \cdot 2$ & $5 \cdot 4$ & \\
\hline BAC & 1.5 & NR & & $1 \cdot 5$ & $1 \cdot 8$ & \\
\hline Carcinoma NOS & $28 \cdot 8$ & $27 \cdot 6$ & & $28 \cdot 8$ & $30 \cdot 1$ & \\
\hline \multicolumn{3}{|c|}{ Charlson Score (\%) } & 0.01 & & & 0.70 \\
\hline 0 & $63 \cdot 9$ & $68 \cdot 0$ & & $64 \cdot 2$ & $61 \cdot 9$ & \\
\hline 1 & $22 \cdot 9$ & $21 \cdot 3$ & & $22 \cdot 8$ & $23 \cdot 4$ & \\
\hline
\end{tabular}




\begin{tabular}{|c|c|c|c|c|c|c|}
\hline Variables & $\begin{array}{l}\text { Carboplatin-based } \\
\text { Doublet } N=12,501\end{array}$ & \multicolumn{2}{|c|}{$\begin{array}{l}\text { Cisplatin-based } \\
\text { Doublet } N=905\end{array}$} & $\begin{array}{l}\text { Carboplatin-based } \\
\text { Doublet } N=12,501\end{array}$ & \multicolumn{2}{|c|}{ Cisplatin-based Doublet N=905 } \\
\hline 2 & $8 \cdot 0$ & $7 \cdot 6$ & & $7 \cdot 9$ & $8 \cdot 7$ & \\
\hline $3+$ & $5 \cdot 2$ & $3 \cdot 1$ & & $5 \cdot 1$ & $6 \cdot 1$ & \\
\hline COPD & & & 0.04 & & & 0.72 \\
\hline DX only & $39 \cdot 8$ & $40 \cdot 8$ & & $39 \cdot 8$ & $40 \cdot 5$ & \\
\hline Hosp/ER & $3 \cdot 6$ & $2 \cdot 0$ & & $3 \cdot 5$ & $2 \cdot 8$ & \\
\hline \multicolumn{7}{|c|}{ Care in Prior $\mathrm{Yr}$} \\
\hline Any Inpatient & $56 \cdot 5$ & $56 \cdot 2$ & 0.89 & $56 \cdot 3$ & $59 \cdot 4$ & $0 \cdot 15$ \\
\hline Hospital days & $5 \cdot 9$ & $5 \cdot 4$ & 0.03 & $5 \cdot 9$ & $6 \cdot 1$ & 0.62 \\
\hline Home health & $7 \cdot 5$ & $6 \cdot 6$ & $0 \cdot 34$ & $7 \cdot 4$ & $6 \cdot 8$ & 0.60 \\
\hline SNF & $4 \cdot 8$ & $2 \cdot 8$ & $<0.01$ & $4 \cdot 7$ & $6 \cdot 8$ & $0 \cdot 14$ \\
\hline Supplemental Oxygen (\%) & $22 \cdot 0$ & $17 \cdot 7$ & $<0.01$ & $21 \cdot 7$ & $22 \cdot 4$ & 0.70 \\
\hline Assistive Devices* (\%) & $11 \cdot 6$ & $9 \cdot 2$ & 0.03 & $11 \cdot 5$ & $10 \cdot 5$ & 0.48 \\
\hline Brain Metastases (\%) & $14 \cdot 4$ & $11 \cdot 4$ & 0.01 & $14 \cdot 2$ & $15 \cdot 2$ & 0.54 \\
\hline \multicolumn{3}{|c|}{ Yr of Diagnosis } & $<0.01$ & & & 0.19 \\
\hline 1995 & $0 \cdot 8$ & $3 \cdot 7$ & & $0 \cdot 8$ & $1 \cdot 8$ & \\
\hline 1996 & $1 \cdot 5$ & $3 \cdot 5$ & & 1.6 & $1 \cdot 5$ & \\
\hline 1997 & 1.9 & $4 \cdot 0$ & & $2 \cdot 0$ & 1.9 & \\
\hline 1998 & $2 \cdot 2$ & $2 \cdot 4$ & & $2 \cdot 2$ & $2 \cdot 8$ & \\
\hline 1999 & $2 \cdot 8$ & $2 \cdot 5$ & & $2 \cdot 7$ & $4 \cdot 1$ & \\
\hline 2000 & $6 \cdot 7$ & $3 \cdot 9$ & & $6 \cdot 5$ & $8 \cdot 2$ & \\
\hline 2001 & $7 \cdot 8$ & $6 \cdot 1$ & & $7 \cdot 7$ & $7 \cdot 6$ & \\
\hline 2002 & 8.0 & $5 \cdot 5$ & & $7 \cdot 9$ & $7 \cdot 5$ & \\
\hline 2003 & $9 \cdot 8$ & $7 \cdot 5$ & & $9 \cdot 7$ & $9 \cdot 1$ & \\
\hline 2004 & $11 \cdot 3$ & $10 \cdot 1$ & & $11 \cdot 3$ & $12 \cdot 1$ & \\
\hline 2005 & $11 \cdot 1$ & $8 \cdot 8$ & & $10 \cdot 9$ & $9 \cdot 5$ & \\
\hline 2006 & $10 \cdot 3$ & $9 \cdot 1$ & & $10 \cdot 2$ & 8.9 & \\
\hline 2007 & $9 \cdot 3$ & $10 \cdot 3$ & & $9 \cdot 4$ & $9 \cdot 3$ & \\
\hline 2008 & $9 \cdot 1$ & $10 \cdot 7$ & & $9 \cdot 4$ & $6 \cdot 9$ & \\
\hline 2009 & $7 \cdot 5$ & $11 \cdot 9$ & & $7 \cdot 7$ & $8 \cdot 7$ & \\
\hline \multicolumn{3}{|c|}{ SEER-Region (\%) } & $<0.01$ & & & $1 \cdot 00$ \\
\hline Atlanta & $4 \cdot 0$ & NR & & $3 \cdot 9$ & $4 \cdot 1$ & \\
\hline Connecticut & $7 \cdot 5$ & $5 \cdot 6$ & & $7 \cdot 4$ & $7 \cdot 2$ & \\
\hline Detroit & $8 \cdot 7$ & $14 \cdot 8$ & & $9 \cdot 1$ & $9 \cdot 0$ & \\
\hline Greater GA & $8 \cdot 8$ & $5 \cdot 0$ & & 8.6 & $9 \cdot 0$ & \\
\hline Greater CA & $15 \cdot 0$ & $18 \cdot 8$ & & $15 \cdot 2$ & $15 \cdot 6$ & \\
\hline Hawaii & $1 \cdot 2$ & NR & & $1 \cdot 2$ & $1 \cdot 7$ & \\
\hline lowa & $6 \cdot 8$ & $6 \cdot 7$ & & $6 \cdot 8$ & $6 \cdot 4$ & \\
\hline Kentucky & $8 \cdot 2$ & $7 \cdot 0$ & & $8 \cdot 1$ & $9 \cdot 0$ & \\
\hline Louisiana & $6 \cdot 1$ & $5 \cdot 1$ & & $6 \cdot 0$ & $5 \cdot 3$ & \\
\hline New Jersey & $12 \cdot 2$ & $10 \cdot 9$ & & $12 \cdot 1$ & $11 \cdot 4$ & \\
\hline New Mexico & $1 \cdot 7$ & $2 \cdot 0$ & & $1 \cdot 7$ & 1.9 & \\
\hline Seattle & $6 \cdot 5$ & $5 \cdot 9$ & & $6 \cdot 5$ & $5 \cdot 9$ & \\
\hline LA & $5 \cdot 6$ & $8 \cdot 8$ & & $5 \cdot 8$ & $5 \cdot 8$ & \\
\hline San Jose & $2 \cdot 3$ & $3 \cdot 3$ & & $2 \cdot 4$ & $2 \cdot 4$ & \\
\hline San Francisco & $3 \cdot 9$ & $2 \cdot 2$ & & $3 \cdot 8$ & $4 \cdot 1$ & \\
\hline Utah & $1 \cdot 5$ & 1.6 & & $1 \cdot 5$ & $1 \cdot 3$ & \\
\hline
\end{tabular}

NSCLC: Non-Small Cell Lung Cancer; BAC: Bronchoaveolar Carcinoma; NOS: Not Otherwise Specified; SNF: Skilled Nursing Facility; DX: Diagnosis; COPD: Chronic Obstructive Pulmonary Disease; ER: Emergency Room; O2: Oxygen; YR: year; MOS: Months; NR: Not Reported to Protect Identity of Patients within Cell Sizes Less than 11.

results to predict each patient's probability of receiving a cisplatinum-based regimen based on their observed characteristics to compute a propensity score (PS) weight. Specifically, for cisplatin patients their PS weight was equal to 1/PS and the PS weight was equal to $1 /(1-\mathrm{PS})$ for carboplatin patients. Observed characteristics were very similar in the two treatment groups after applying these PS weights (Table 1). We then compared survival and time to first hospitalization in the two cohorts using 
weighted Kaplan-Meier curves and weighted Cox regression models. We also computed weighted morbidity outcomes in the two cohorts and tested for differences using Chi-square or T-tests that accounted for weighting. Two-sided $p$ values of $<0.05$ were considered statistically significant. To provide estimates of the effect of chemotherapy type on mortality and morbidity according to key patient factors, including patient age and lung cancer history, we fit weighted Cox models within patient strata $[30,31]$

\section{Results}

As shown in Table 1 (left columns), the NSCLC patients in the cisplatin-based doublet and carboplatin-based doublet cohorts varied with respect to prognostically important patient demographic and disease attributes prior to propensity score weighting. Specifically, patients in the cisplatin-based doublet cohort were, on average, younger, more often male, and more often married. They were also less likely to have diagnosis codes for brain metastases, had lesser amounts of comorbid disease including COPD, and were less likely to be using oxygen. They had less health care utilization in the year prior to the initiation of chemotherapy as well including lower rates of use of hospitals, SNF stays, and functional assistance devices. Finally, use of cisplatin-based doublets increased over the study period and varied substantially across geographic areas. After implementation of propensity score weighting however, the attributes of NSCLC patients in the cisplatin-based doublet and carboplatin-based doublet cohorts were very similar (Table 1, right columns).

In Table 1, Comparison of attributes of elderly Medicare patients with NSCLC who were treated with either a cisplatin-based doublet chemotherapy regimen or a carboplatin-based doublet chemotherapy regimen before and after propensity score weighting.
Table $\mathbf{2}$ and Figure 1 show that after propensity score weighting, the survival estimates for the NSCLC patients were slightly better in patients treated with cisplatin-based doublets compared with those treated with carboplatin-based doublets. The median survival for cisplatin-based doublet patients was 7.4 months and for carboplatin-based doublet patients 7.0 months $(p=0.05)$. The subset of NSCLC patients with adenocarcinoma histology had median survivals of 7.6 months for both types of regimens (Table 2).

In Figure 1, Survival time plotted using the Kaplan-Meier (K-M) method in months for elderly Medicare patients with stage IV non-small cell lung cancer (NSCLC) following treatment with either a cisplatin-based doublet chemotherapy regimen or a carboplatin-based doublet regimen. The log-rank test is used to test the hypothesis that the two survival distributions are equal. Observations were censored at five years and numbers at risk are after propensity score weighting.

Similarly, there was no difference in survival for patients with squamous cell histology. Patients with carcinoma not otherwise specified (NOS) had a survival benefit associated with cisplatinbased doublets (median survival 8.1 months vs. 6.5 months, $\mathrm{P}=0.01$ ). Figure $\mathbf{2}$ is a forest plot showing that the relative advantage of cisplatin-based doublets over carboplatin-based doublets attenuates with age and is only statistically significant among the youngest patients studied: those 66 through 69 years of age. In this forest plot (Figure 2), unity (1.0) represents equality in patient survival between carboplatin-based vs. cisplatin-based doublet therapy among elderly Medicare patients with metastatic NSCLC. Deviation rightward represents superior survival associated with cisplatin-based doublet therapies and deviation leftward represents superior survival associated with carboplatin-based doublet therapies. NSCLC=non-small cell lung cancer.

Mortality following treatment expressed in survival time in

Table 2 Association between carboplatin- vs. cisplatin -based doublet chemotherapy regimens for metastatic NSCLC after propensity score weighting and survival outcomes by tumor histology and patient age $(\mathrm{N}=13,406)$.

\begin{tabular}{|c|c|c|c|c|c|c|c|}
\hline \multirow[t]{2}{*}{ Variables } & $\begin{array}{c}\text { Carboplatin } \\
\text { Doublet }\end{array}$ & Cisplatin Doublet & $\begin{array}{c}\text { Carboplatin } \\
\text { Doublet }\end{array}$ & Cisplatin Doublet & & \multicolumn{2}{|c|}{$\begin{array}{c}\text { Hazard of Death Associated with } \\
\text { Carboplatin- vs. Cisplatin-Based } \\
\text { Doublet Treatment }\end{array}$} \\
\hline & \multicolumn{2}{|r|}{$\mathbf{N}$} & \multicolumn{2}{|c|}{ Median Survival (months) } & p-value & HR & $(95 \% \mathrm{Cl})$ \\
\hline All & 12,501 & 905 & $7 \cdot 0$ & $7 \cdot 4$ & 0.05 & 1.08 & (0.99 to 1.18$)$ \\
\hline Large Cell & 642 & 50 & $6 \cdot 1$ & $4 \cdot 6$ & 0.76 & 1.06 & (0.74 to 1.52$)$ \\
\hline Carcinoma NOS & 3604 & 250 & $6 \cdot 5$ & $8 \cdot 1$ & 0.01 & $1 \cdot 21$ & (1.04 to 1.41$)$ \\
\hline Adenocarcinoma & 5265 & 363 & $7 \cdot 6$ & $7 \cdot 6$ & 0.52 & 1.05 & $(0.91-1.20)$ \\
\hline Squamous Cell & 2801 & 232 & $7 \cdot 1$ & $6 \cdot 7$ & 0.55 & 1.05 & $(0.89$ to $1 \cdot 24)$ \\
\hline BAC & 189 & 10 & $7 \cdot 5$ & $1 \cdot 7$ & 0.75 & 0.89 & $(0.42$ to 1.87$)$ \\
\hline $66-69$ & 3,594 & 324 & $7 \cdot 2$ & $8 \cdot 0$ & $<0.01$ & $1 \cdot 22$ & (1.07 to 1.39$)$ \\
\hline $70-74$ & 4,292 & 360 & $7 \cdot 0$ & $7 \cdot 6$ & $0 \cdot 14$ & $1 \cdot 10$ & (0.97 to 1.25$)$ \\
\hline $75-79$ & 3,319 & 174 & $6 \cdot 8$ & $7 \cdot 0$ & 0.55 & 1.06 & (0.88 to $1 \cdot 28)$ \\
\hline $80-84$ & 1,239 & 41 & $7 \cdot 1$ & $3 \cdot 7$ & 0.56 & 0.89 & (0.60 to 1.32$)$ \\
\hline$>85$ & 237 & 6 & $6 \cdot 3$ & $6 \cdot 1$ & 0.69 & 0.87 & (0.45 to 1.70$)$ \\
\hline
\end{tabular}

NSCLC=Non-Small Cell Lung Cancer; HR=Hazard Ratio; Cl=Confidence Interval, BAC=bronchoaveolar carcinoma; NOS=not otherwise specified. 

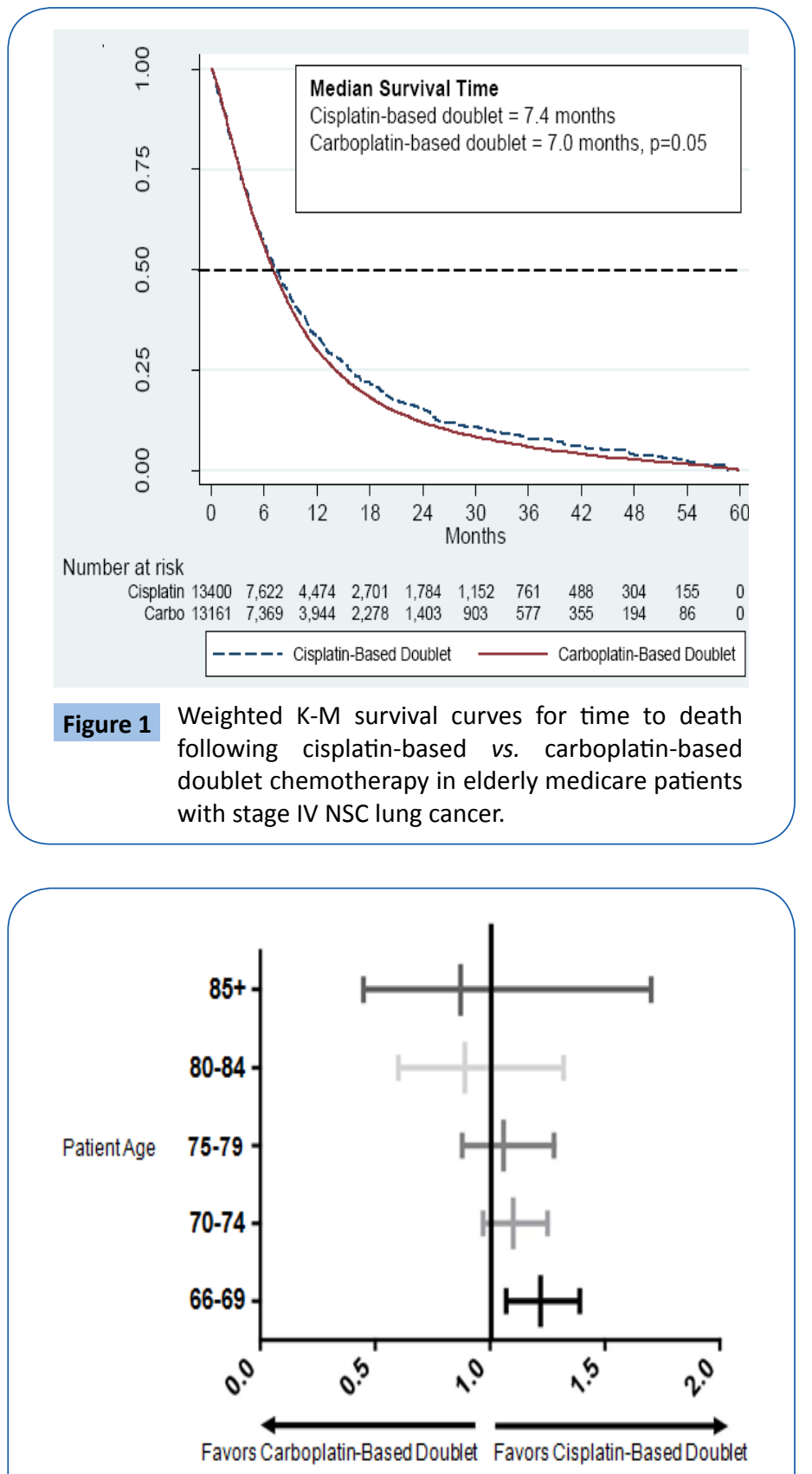

Figure 2 Forest plot comparing relative hazard of death of carboplatin- vs. cisplatin-based doublets in elderly medicare patients with advanced NSCLC by patient age $(\mathrm{N}=13,406)$.

months and hazard ratio measured from first day of treatment with chemotherapy through death or fixed right censoring for elderly Medicare patients with NSCLC who were treated with either a cisplatin-based doublet chemotherapy regimen or a carboplatin-based doublet chemotherapy regimen according to patient age at treatment initiation and NSCLC tumor histology in Table 2.

Table 3 shows that patients treated with doublet therapy have high use of medical services following initiation of chemotherapy, with higher adjusted rates of service use among patients treated with cisplatin-based doublet regimens compared with patients treated with carboplatin-based doublet regimens. Specifically, patients treated with cisplatin-based doublets were more likely than those treated with carboplatin-based doublets to be hospitalized $(84.8 \%$ vs. $80.6 \%, p=0.01)$, had a higher mean number of hospital admissions ( 2.2 vs. $1.8, p<0.01)$, longer mean lengths of hospital stay among those hospitalized ( 16.9 days vs. 14.4 days, <0.01), and longer mean number of ICU days among those admitted to an ICU ( 2.2 days vs. 1.8 days, $p<0.01$ ). Patients treated with cis- versus carboplatin-based regimens also had more hospitalizations with discharge to a location other than their home (1.2 vs. 1.0 admissions, $\mathrm{p}=0.02$ ). In Table 3, Hospital-based health care utilization is the time from the first day of treatment with chemotherapy through death or fixed right censoring for elderly Medicare patients with NSCLC who were treated with either a cisplatin-based doublet chemotherapy regimen or a carboplatin-based doublet chemotherapy regimen.

Figure 3 is a forest plot showing that the youngest patients studied, those 66 through 69 years of age, did not experience excess hospitalizations in the setting of treatment with cisplatin-based doublet chemotherapy regimens relative to carboplatin-based regimens. Patients aged $70-74$ and $75-79$ receiving carboplatin-

Table 3: Association between carboplatin- vs. cisplatin -based doublet chemotherapy regimens for metastatic NSCLC after propensity score weighting and hospital-based health care utilization $(N=13,406)$.

\begin{tabular}{|c|c|c|c|}
\hline \multirow[t]{2}{*}{ Variables } & $\begin{array}{c}\text { Carboplatin- } \\
\text { Based Doublet }\end{array}$ & $\begin{array}{c}\text { Cisplatin- } \\
\text { Based Doublet }\end{array}$ & \multirow[t]{2}{*}{ p-value } \\
\hline & 12,501 & 905 & \\
\hline Any ER Visit (\%) & $92 \cdot 7$ & $93 \cdot 4$ & $0 \cdot 51$ \\
\hline Any Inpatient (\%) & $80 \cdot 6$ & $84 \cdot 8$ & $0 \cdot 01$ \\
\hline Any ICU (\%) & $27 \cdot 4$ & $30 \cdot 7$ & 0.09 \\
\hline Any CCU (\%) & $12 \cdot 3$ & $13 \cdot 7$ & $0 \cdot 28$ \\
\hline Any Intubation (\%) & $6 \cdot 3$ & $7 \cdot 1$ & 0.49 \\
\hline $\begin{array}{l}\text { Any Hospital Discharge to } \\
\text { Non- Home Location (\%) }\end{array}$ & $64 \cdot 1$ & $67 \cdot 9$ & 0.06 \\
\hline Any home health (\%) & $42 \cdot 6$ & $45 \cdot 4$ & $0 \cdot 21$ \\
\hline Any SNF (\%) & $18 \cdot 7$ & $20 \cdot 4$ & $0 \cdot 37$ \\
\hline Any Hospice (\%) & $48 \cdot 6$ & $45 \cdot 7$ & $0 \cdot 19$ \\
\hline Death in Hospital (\%) & $22 \cdot 6$ & $23 \cdot 8$ & 0.49 \\
\hline New Hemodialysis (\%) & 0.4 & $1 \cdot 2$ & 0.94 \\
\hline ER Visits (mean) & $4 \cdot 2$ & $4 \cdot 5$ & $0 \cdot 13$ \\
\hline $\begin{array}{l}\text { Hospital Admissions } \\
\text { (mean) }\end{array}$ & 1.9 & $2 \cdot 2$ & $<0.01$ \\
\hline Inpatient LOS (mean) & $11 \cdot 6$ & $14 \cdot 3$ & $<0.01$ \\
\hline $\begin{array}{l}\text { Inpatient LOS Among } \\
\text { Admitted (mean) }\end{array}$ & $14 \cdot 4$ & $16 \cdot 9$ & $<0.01$ \\
\hline ICU LOS(mean) & $1 \cdot 8$ & $2 \cdot 2$ & 0.04 \\
\hline $\begin{array}{l}\text { ICU LOS Among Admitted } \\
\text { (mean) }\end{array}$ & $6 \cdot 4$ & $7 \cdot 1$ & $0 \cdot 19$ \\
\hline CCU LOS (mean) & $0 \cdot 7$ & 0.9 & $0 \cdot 11$ \\
\hline $\begin{array}{l}\text { CCU LOS Among Admitted } \\
\text { (mean) }\end{array}$ & $5 \cdot 5$ & $6 \cdot 2$ & $0 \cdot 21$ \\
\hline $\begin{array}{c}\text { Hospital Discharges to } \\
\text { Non-home Location (mean) }\end{array}$ & $1 \cdot 0$ & $1 \cdot 1$ & 0.02 \\
\hline $\begin{array}{l}\text { Hospice LOS Among } \\
\text { Patients Enrolled (mean) }\end{array}$ & $14 \cdot 1$ & $15 \cdot 1$ & $0 \cdot 15$ \\
\hline
\end{tabular}

NSCLC: Non-Small Cell Lung Cancer; LOS: Length of Stay; D/C: Discharge 


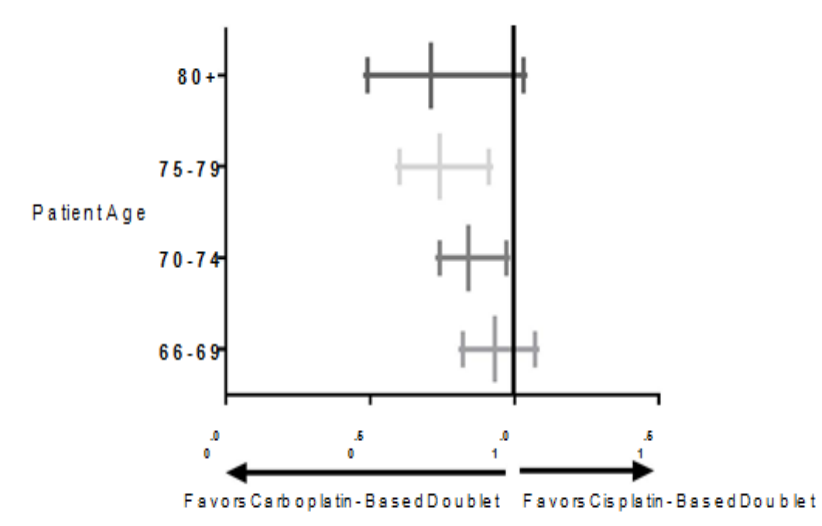

Figure 3 Forest plot comparing relative hazard of hospitalization for carboplatin- vs. cisplatin-based doublets in elderly medicare patients with advanced NSCLC by patient age $(\mathrm{N}=13,406)$.

based versus cisplatin-based regimens had statistically significant reductions in the risk of hospitalization, although the risk reduction was not significant in the oldest, patients (i.e., those who were $>80$ years of age), thought the $95 \%$ confidence interval is understandably wide with only 47 patients in the cisplatinbased doublet group. In this forest plot (Figure 3), unity (1.0) represents equality in patient survival between carboplatin-based vs. cisplatin-based doublet therapy among elderly Medicare patients with metastatic NSCLC. Deviation rightward represents a longer time to first hospitalization associated with cisplatinbased doublet therapies and deviation leftward represents a longer time to first hospitalization associated with carboplatinbased doublet therapies (NSCLC=non-small cell lung cancer).

\section{Discussion}

Our results show that overall there was a clinically small survival advantage of marginal statistical significance for elderly Medicare patients with NSCLC who are treated with cisplatinbased doublet chemotherapy regimens compared to patients treated with carboplatin-based doublet chemotherapy regimens (7.4 vs. 7.0 months, $p=0.05$ ). However, the overall survival benefit appears to have been due to a larger survival advantage among the large subset of patients aged 66 through 69 years who lived approximately three and one half weeks longer than similarly aged patients treated with carboplatin-based doublet regimens $(p<0.01)$. There was no apparent cisplatin-associated survival advantage among patients 70 years and older. Among this latter group of patients, there were clinically and statistically meaningful differences in morbidity associated with cisplatinbased therapy. Patients who were treated with cisplatin-based doublet chemotherapy regimens were observed to use more (1) hospital-based health care and (2) more intensive hospitalbased care following the beginning of chemotherapy than patients who were treated with carboplatin-based doublets. These results advance clinical medicine in several ways. First and most importantly, we fill an existing void by providing "real world" estimates of morbidity and mortality outcomes in elderly Medicare patients with NSCLC according to cisplatin-based vs. carboplatin-based doublet therapy and according to tumor histology and patient age. These results may be immediately useful to oncologists and their older patients as they discuss treatment options by providing more representative estimates of morbidity and mortality of two first-line treatment options. These real world estimates are of critical importance given that the average survival following both forms of doublet therapies (i.e., 7.0 months for carboplatin-based doublets to 7.4 months for cisplatin-based doublets) were approximately six weeks shorter than those reported in existing data like meta-analyses of clinical trials (i.e., 8.4 to 9.1 months respectively) [11]. Further, the small ( $<2$ weeks) and marginally significant survival advantage associated with cisplatin-based regimens in the larger cohort was clearly driven by the youngest patients, those 66-69 years of age who experienced a larger ( $>3$ weeks) survival advantage which was statistically significant. For those 70 years of age and older, there was no survival advantage, but clearly greater morbidity as represented by greater subsequent use and intensity of hospitalbased health care. This suggests that carboplatin-based doublets are superior to cisplatin-based doublets for such patients through of minimization of treatment-related morbidity.

Limitations to the work include the fact that we rely on administrative data that do not contain information about patient or physician preferences in a setting in which treatments were not randomly allocated. For example, our measure of patient toxicity or morbidity is hospital-based health care use. While we have previously shown that hospital-based health care use is a good proxy for chemotherapy-related toxicity among patients with colon and breast cancer treated on clinical trials, we are extrapolating those findings to patients with NSC lung cancer treated off clinical trials [26]. In addition, we studied patients living in SEER areas only; generalizability of our findings to other areas requires further study. In order to identify chemotherapy regimens, we select patients who begin chemotherapy in the ambulatory setting. This is related to how chemotherapy is coded when administered in the hospital (no detail about the exact drugs are discernable) vs. the ambulatory where J codes reliably represent even multidrug regimens [32].

However, this mean, our results are generalizable to patients who are fit enough to begin chemotherapy in the ambulatory setting, not the hospital. Finally, as others have found, we found that patients with the diagnosis of carcinoma NOS was substantial and more likely among those diagnosed via cytology rather than pathology (results not shown) $[33,34]$. These patients were among the subsets of patients for whom cisplatin-based doublets were clearly advantageous in our results, results we have not seen reported elsewhere. It is possible that some percentage of the NOS patients had small cell lung cancer lung cancer, a disease which is rapidly fatal when untreated, but for which cisplatinbased therapy has previously been shown to be associated with survivals of nine months or more in the usual care setting, which is two months longer than patients treated for NSCLC [35]. 


\section{Conclusion}

Comparing first-line platinum-based doublet chemotherapy regimens administered to elderly Medicare patients with stage IV NSCLC, we conclude that if treatment with a platinum-based doublet is being considered by a patient and their oncologist, it is reasonable to consider cisplatin-based doublet chemotherapy regimens for patients who are aged 66-69 and are willing and able to accept the risks associated with cisplatin-based therapy. However, if a platinum-based doublet is being considered by patients who are $>70$ years of age and their oncologists, carboplatin should be the platinum backbone of the regimen rather than cisplatin as the latter confers increased toxicity without improvement in survival.

\section{Contributors}

There are no contributors beyond the authors.

\section{Author Specific Contributions}

Elizabeth Lamont: Literature search, figures, study design, data collection, data interpretation, and writing.

Nancy L Keating: Data interpretation, and writing.

\section{References}

1 National Cancer Institute: Surveillance, Epidemiology and End Results Program (2014) Cancer stat fact: Lung and bronchus cancer.

2 Non-small cell lung cancer collaborative group (1995) Chemotherapy in non-small cell lung cancer: A meta-analysis using updated data on individual patients from 52 randomized trials. BMJ 311: 899-909.

3 Le Chevalier T, Brisgand D, Douillard JY, Pujol JL, Alberola V, et al. (1994) Randomized study of vinorelbine and cisplatin versus vindesine and cisplatin versus vinorelbine alone in advanced nonsmall-cell lung cancer: Results of a European multicenter trial including 612 patients. J Clin Oncol 12: 360-367.

4 Wozniak AJ, Crowley JJ, Balcerzak SP (1996) Randomized phase III trial of cisplatin (CDDP) vs. CDDP plus navelbine (NVB) in treatment of advanced non-small cell lung cancer (NSCLC): Report of a southwest oncology group study (SWOG-9308). Proc ASCO 15: 374.

5 NSCLC Meta-analyses Collaborative Group (2008) Chemotherapy in addition to supportive care improves survival in advanced non-small cell lung cancer: A systematic review and meta-analysis of individual patient data from 16 randomized controlled trials. J Clin Oncol 26 : 4617-4625.

6 Schiller JH, Harrington D, Belani CP, Langer C, Sandler A, et al. (2002) Comparison of four chemotherapy regimens for advanced nonsmall-cell lung cancer. N Engl J Med 346: 92-98.

7 Chen YM, Perng RP, Tsai CM, Whang-Peng J (2006) A phase II randomized study of paclitaxel plus carboplatin or cisplatin against chemo-naïve inoperable non-small cell lung cancer in the elderly. J Throac Oncol 1: 141-145.

8 Langer C, Li S, Schiller J, Tester W, Rapoport BL, et al. (2007) Randomized phase II trial of paclitaxel plus carboplatin gemcitabine plus cisplatin in eastern cooperative oncology group performance status 2 non-small-cell lung cancer patients: ECOG 1599. J Clin Oncol 25: 418-423.
Christopher Azzoli: Literature search, data interpretation, and writing.

Mary Beth Landrum: Study design, data analysis, data interpretation, and writing.

\section{Funding}

This work was supported by grants from the National Institutes of Health (grant number R01CA142744 to Landrum, R01CA132900 to Lamont). The funding source had no role in the design, analyses, interpretation of results, nor writing of manuscript. The funding source allowed purchase of data, programmers' time, and investigators' time.

\section{Declaration of Interests}

The authors declare no conflicts of interest.

\section{Acknowledgements}

We gratefully acknowledge project support from Lauren Riedel which includes research assistance, tabular displays, manuscript proofreading, and manuscript formatting and submission. Additionally, we are grateful to Ms. Christina Fu for excellent data programming support.

9 Jiang J, Liang X, Zhou X, Huang R, Chu Z (2007) A meta-analysis of randomized controlled trials comparing carboplatin-based to cisplatin-based chemotherapy in advanced non-small cell lung cancer. Lung Cancer 57: 348-358.

10 Rosell R, Gatzemeier U, Betticher DC, Keppler U, Macha HN, et al. (2002) Phase III randomized trial comparing paclitaxel/carboplatin with paclitaxel/cisplatin in patients with advanced non-small-cell lung cancer: A cooperative multinational trial. Ann Oncol 13: 15391549.

11 Ardizzoni A, Boni L, Tiseo M, Fossella FV, Schiller JH, et al. (2002) Cisplatin- versus carboplatin-based chemotherapy in first-line treatment of advanced non-small-cell lung cancer: An individual patient data meta-analysis. J Natl Cancer Inst 99: 847-57.

12 de Castria TB, da Silva EMK, Gois AFT, Riera R (2013) Cisplatin versus carboplatin in combination with third-generation drugs for advanced non-small cell lung cancer. Cochrane Database Syst Rev 8: CD009256.

13 Luo J, Leaw SJ, Xu Y, Zheng D (2011) Comparison of cisplatin- and carboplatin-based third-generation chemotherapy in 1,014 Chinese patients with advanced non-small-cell lung cancer. Med Oncol 28: 1418-1424.

14 Earle CC, Tsai JS, Gelber RD, Weinstein MC, Neumann PJ, et al. (2001) Effectiveness of chemotherapy for advanced lung cancer in the elderly: Instrumental variable and propensity analysis. J Clin Oncol 19: 1064-1070.

15 Davidoff AJ, Tang M, Seal B, Edelman MJ (2010) Chemotherapy and survival benefit in elderly patients with advanced non-small-cell lung cancer. J Clin Oncol 28: 2197-2197.

16 Santana-Davila R, Szabo A, Arce-Lara C, Williams CD, Kelley MJ, et al. (2014) Cisplatin versus carboplatin-based regimens for the treatment of patients with metastatic lung cancer. An analysis of Veterans Health Administration data. J Thorac Oncol 9: 702-709. 
17 Landrum MB, Keating NL, Lamont EB, Bozeman SR, Krasnow SH, et al. (2012) Survival of older patients with cancer in the veterans' health administration versus fee-for-service Medicare. J Clin Oncol 30: 1072-1079.

18 Lamont EB, Landrum MB, Keating NL, Archer L, Lan L, et al. (2010) Differences in clinical trial patients' attributes and outcomes according to enrollment setting. J Clin Oncol 28:215-221.

19 Agha Z, Lofgren RP, Van Ruiswyk JV, Layde PM (2000) Are patients at Veterans Affairs medical centers sicker? A comparative analysis of health status and medical resource use. Arch Intern Med 160: 3252.

20 Nelson KM (2006) The burden of obesity among a national probability sample of Veterans. J Gen Intern Med 21: 915-919.

21 Kazis LE, Miller DR, Clark J, Skinner K, Lee A, et al. (1998) Healthrelated quality of life in patients served by the department of veterans affairs: Results from the veterans health study. Arch Intern Med 158: 626-632.

22 Ries LAG, Kosary CL, Hankey BF, Miller BA, Harras A, et al. (1997) SEER cancer statistics review, 1973-1994. Bethesda, National Cancer Institute.

23 National Cancer Institute: Surveillance, Epidemiology, and End Results Program (2014) Overview of the Seer Program.

24 Nattinger AB, McAuliffe TL, Schapira MM (1997) Generalizability of the surveillance, epidemiology and end results registry population: Factors relevant to epidemiologic and health care research. J Clin Epidemiol 50: 939-945.

25 Lamont EB, Lan L (2014) Sensitivity of Medicare claims data for measuring use of standard multi-agent chemotherapy regimens. Medical Care 52: e15-20.

26 Lamont EB, He Y, Saltz L, Muss H, Zaslavsky AM (2014) Hospital- based health care use correlates with incidence of treatment-related adverse events among elderly Medicare patients treated on adjuvant chemotherapy trials (Alliance 70802). J Geriatr Oncol 5: 230-237.

27 Eichler AF, Lamont EB (2009) Utility of administrative claims data for the study of brain metastases: a validation study. J Neurooncol 95: 427-431.

28 Rosenbaum PR, Rubin DB (1984) Reducing bias in observational studies using subclassification on the propensity score. J Am Stat Assoc 79: 516-524.

29 Rosenbaum PR, Rubin DB (1984) The central role of the propensity score in observational studies for causal effects. Biometrika 70: 41-55.

30 Rosenbaum PR, Rubin DB (1985) Constructing a control group using multivariate matched sampling methods that incorporate the propensity score. Am Stat 39: 33-38.

31 Rubin DB, Thomas N (1996) Matching using estimated propensity scores: Relating theory to practice. Biometrics 52: 249-64.

32 Smith HL (1997) Matching with multiple controls to estimate treatment effects in observational studies. Sociol methodol: 325-353.

33 Sagerup CM, Smastuen M, Johannesen TB, Helland A, Brustugun OT (2012) Increasing age and carcinoma not other specified: A 20-year population study of 40,118 lung cancer patients. J Thorac Oncol 7: 57-63.

34 Ou SH, Zell JA (2009) Carcinoma NOS is a common histologic diagnosis and is increasing in proportion among non-small cell lung cancer histologies. J Thorac Oncol 4: 1202-1211.

35 Lamont EB, Schilsky RL, He Y, Muss H, Cohen H, et al. (2015) Generalizability of trial results to elderly Medicare patients with advanced solid tumors (Alliance 70802). J Natl Cancer Inst 107: 1-7. 